\title{
POTENCIAL DO ÓXIDO DE FERRO COMO MARCADOR FECAL EM OVINOS
}

\author{
Priscila de Souza Ramos ${ }^{1}$ \\ Bruno Cabral Soares ${ }^{2}$ \\ Maria de Fátima Araújo Vieira ${ }^{3}$ \\ Alaire Franco Tavares ${ }^{4}$ \\ Ana Joyce Torres De Oliveira ${ }^{4}$ \\ Andreza Araujo Cardoso ${ }^{4}$ \\ Arlison Santos dos Santos ${ }^{4}$ \\ Henrique da Silva Dias ${ }^{4}$ \\ Isabela Brasil Ferreira ${ }^{4}$ \\ João Paulo Sousa Silva ${ }^{4}$ \\ Pedro Henrique do Nascimento ${ }^{4}$ \\ Juliana Abreu Silva ${ }^{4}$ \\ Rafael Aquino de Oliveira ${ }^{4}$ \\ Leticia de Abreu Faria ${ }^{3}$
}

\begin{abstract}
RESUMO
Os marcadores são ferramentas que auxiliam a obtenção de informações que aprimoram a produção animal, principalmente em nutrição. Objetivou-se avaliar doses de óxido de ferro como marcador fecal e seus efeitos na coloração das fezes de ovinos. O delineamento experimental foi em quadrado latino 4 x 4, compreendendo quatro doses de adição de óxido de ferro $\left(\mathrm{Fe}_{2} \mathrm{O}_{3}\right)$ de $0,5,10$ e $20 \%$ do concentrado, equivalentes a $0,7,5,15,30 \mathrm{~g}$ animal $^{-1}$, respectivamente. Foram utilizados quatro ovinos machos não castrados mantidos em gaiolas metabólicas adaptadas, alimentados com dieta com relação volumoso: concentrado de 84: 16, sendo feno de Tifton 85 triturado e o suplemento energético a base de milho moído. $\mathrm{O}$ período experimental teve duração de 47 dias, contabilizando 15 dias para adaptação, sendo que cada subperíodo compreendeu 7 dias mais um de descanso. Os animais receberam os tratamentos com as doses de $\mathrm{Fe}_{2} \mathrm{O}_{3}$ misturadas ao concentrado no primeiro dia de cada subperíodo experimental e seguia-se as coletas de fezes total diariamente diretamente da gaiola. Não foram verificados efeitos dos tratamentos no consumo voluntário de matéria seca, comportamento ingestivo e total de fezes. A coloração das fezes dos animais foi observada em todos os tratamentos a partir das $22 \mathrm{~h}$ e permaneceu sob diferentes intensidades até $130 \mathrm{~h}$, sendo que as doses de 10 e $20 \%$ persistiram até $154 \mathrm{~h}$ após o fornecimento. $\mathrm{O} \mathrm{Fe}_{2} \mathrm{O}_{3}$ tem potencial para uso como marcador fecal, tornando-se uma ferramenta para auxílio na experimentação animal por meio de seus efeitos na coloração das fezes sem alterar o comportamento dos animais. A dose com $10 \%$ de óxido de ferro no suplemento, apresenta maior persistência visual na pigmentação das fezes sem afetar o comportamento de consumo.
\end{abstract}

Palavras-chave: coloração fecal, digestibilidade, excreção fecal, nutrição.

\footnotetext{
${ }^{1}$ Graduanda em Zootecnia, Universidade Federal Rural da Amazônia. Correspondência: priscila.dsr@hotmail.com

${ }^{2}$ Professor da Universidade Federal Rural da Amazônia - UFRA. brunocs@veterinario.med.br

${ }^{3}$ Professora Adjunto da Universidade Federal Rural da Amazônia- UFRA fatima.vieira@ufra.edu.br

${ }^{4}$ Graduandos em Zootecnia, Universidade Federal Rural da Amazônia UFRA
} 


\title{
IRON OXIDE POTENTIAL AS A FECAL MARKER IN SHEEP
}

\begin{abstract}
Markers are tools to support getting information that improves animal production, especially in nutrition. The aim of this study was to evaluate doses of iron oxide as a fecal marker through its effects on fecal coloring of sheep. The experimental design was a 4 x 4 Latin square, comprising four doses of iron oxide $\left(\mathrm{Fe}_{2} \mathrm{O}_{3}\right)$ addition of $0,5,10$ and $20 \%$ of the concentrate, equivalent to $0,7.5,15,30 \mathrm{~g}_{\text {animal }}{ }^{-1}$, respectively. Four non-castrated male sheep were kept in adapted metabolic cages, fed a diet with proportion of forage: concentrate of 84: 16, with crushed Tifton 85 hay and the energy supplement based on ground corn. The experimental period lasted 47 days, counting 15 days for adaptation, with each subperiod comprising 7 days plus one rest. The animals received treatments with $\mathrm{Fe}_{2} \mathrm{O}_{3}$ doses mixed with the concentrate on the first day of each experimental subperiod and total feces collections were followed daily directly from the cage. There were no effects of treatments on voluntary consumption of dry matter, behavior and total feces. The color of the animals' feces was observed in all treatments after $22 \mathrm{~h}$ and remained under different intensities until $130 \mathrm{~h}$, and the doses of 10 and $20 \%$ persisted until $154 \mathrm{~h}$ after supply. The $\mathrm{Fe}_{2} \mathrm{O}_{3}$ has the potential to be used as a fecal marker, becoming a tool to support in animal experimentation through its effects on the color of feces without changing the animals' behavior. The dose with $10 \%$ iron oxide in the supplement, shows greater visual persistence in the pigmentation of feces without affecting the consumption behavior.
\end{abstract}

Keywords: coloring, digestibility, fecal excretion, nutrition.

\section{POTENCIAL DE ÓXIDO DE HIERRO COMO MARCADOR FECAL EN OVEJAS}

\section{RESUMEN}

Los marcadores son herramientas para ayudar a obtener información que mejore la producción animal, especialmente en nutrición. El objetivo de este estudio fue evaluar las dosis de óxido de hierro como marcador fecal a través de sus efectos sobre la coloración fecal de las ovejas. El diseño experimental fue un cuadrado latino $4 \times 4$, que comprende cuatro dosis de adición de óxido de hierro $\left(\mathrm{Fe}_{2} \mathrm{O}_{3}\right)$ de $0,5,10$ y $20 \%$ del concentrado, equivalente a 0 , $7,5,15,30 \mathrm{~g}$ de animal $^{-1}$, respectivamente. Cuatro ovejas macho no castradas fueron mantenidas en jaulas metabólicas adaptadas, alimentadas con una dieta con proporción de forraje: concentrado de 84:16, heno Tifton 85 triturado y el suplemento energético a base de maíz molido. El período experimental duró 47 días, contando 15 días para la adaptación, con cada subperíodo compuesto por 7 días más un descanso. Los animales recibieron tratamientos con dosis de $\mathrm{Fe}_{2} \mathrm{O}_{3}$ mezcladas con el concentrado el primer día de cada subperíodo experimental y las colecciones de heces totales fueron seguidas diariamente directamente desde la jaula. No hubo efectos de los tratamientos sobre el consumo voluntario de materia seca, comportamiento y heces totales. El color de las heces de los animales se observó en todos los tratamientos después de $22 \mathrm{~h}$ y permaneció bajo diferentes intensidades hasta $130 \mathrm{~h}$, y las dosis de 10 y $20 \%$ persistieron hasta $154 \mathrm{~h}$ después del suministro. $\mathrm{El} \mathrm{Fe}_{2} \mathrm{O}_{3}$ tiene el potencial de ser utilizado como un marcador fecal, convirtiéndose en una herramienta para apoyar la experimentación animal a través de sus efectos sobre el color de las heces sin cambiar el comportamiento de los animales. La dosis con $10 \%$ de óxido de hierro en el suplemento, muestra una mayor persistencia visual en la pigmentación de las heces sin afectar el comportamiento de consumo.

Ramos PS, Soares BC, Vieira MFA, Tavares AF, Oliveira AJT, Cardoso AA. et al. Potencial do óxido de ferro como marcador fecal em ovinos. Vet. e Zootec. 2021; v28: 001-013. 
Palabras clave: color, digestibilidad, excreción fecal.

\section{INTRODUÇÃO}

A avaliação de alimentos e alimentação está entre os principais pontos a serem observados no setor de nutrição animal, e devem ocorrer por meio de métodos que visam descrever alimentos e a sua capacidade de sustentar diferentes tipos e níveis de desempenho animal.

Em geral, a maior ênfase é dada à determinação da composição química específica dos alimentos, embora as características físicas também sejam importantes Canesin et al. (1), pois o consumo é fortemente influenciado por inúmeros fatores aliados as características dos alimento ou o ambiente que cerca o animal (2), assim, a avaliação da digestibilidade principalmente in vivo, torna-se o principal alvo de estudos, visando determinar as propriedades inerentes aos alimentos que os tornam passíveis do consumo voluntário.

O consumo voluntário é um dos fatores de elevada relevância quando se trata de ganho em desempenho animal, pois este será o determinante na ingestão de nutrientes para o atendimento das exigências nutricionais, já que serve como um parâmetro para avaliar alimentos, animais e até sistemas de manejo (3).

Uma demanda atual das pesquisas em nutrição animal é a busca de relação entre conteúdo de nutrientes e seu aproveitamento digestivo e metabólico visando respeitar as condições de bem-estar animal, sendo necessário o estabelecimento de métodos apropriados para obtenção de respostas e sucesso na pesquisa (4).

A análise de fezes é uma ferramenta de elevado interesse para a avaliação de alimentos, visando evitar métodos que envolvam procedimentos cirúrgicos (5). No entanto, a coleta de fezes é um desafio em experimentos por muitas vezes causar condições de estresse, seja pelo uso de sacolas ou mesmo pela ingestão forçada de indicadores, além da dificuldade de sua identificação em baias coletivas (6).

Diversos experimentos têm sido conduzidos visando avaliar a efetividade dos marcadores no processo de digestão animal, porém há variabilidade na excreção, dependendo do tipo animal ou da metodologia, fazendo com que as estimativas de produção fecal, consumo e digestibilidade representem menor precisão e acurácia à realidade (7).

$\mathrm{O}$ uso de óxido de ferro $\left(\mathrm{Fe}_{2} \mathrm{O}_{3}\right)$ tem sido usado com suínos como marcador na ração. $\mathrm{O}$ uso de $\mathrm{Fe}_{2} \mathrm{O}_{3}$ como marcador em suínos verificado por meio de abate as 3, 6, 9 e $14 \mathrm{~h}$ após o consumo, a digesta marcada se apresentava, respectivamente, no intestino delgado, intestino grosso, ao longo de todo trato gastrointestinal e excretando digesta marcada, permitindo o estudo da taxa de passagem (8).

$\mathrm{O}$ uso de marcadores para a identificação das fezes promove maior eficiência nos experimentos permitindo avaliar o tempo entre o consumo e a excreção do alimento, o que indica a velocidade de passagem no trato gastrointestinal e pode ser correlacionado à digestibilidade da forragem ou sua combinação com o suplemento.

Diante disso, a hipótese é que o uso de $\mathrm{Fe}_{2} \mathrm{O}_{3}$ tem potencial uso como marcador fecal em ruminantes pelo seu baixo custo, eficiência, facilidade e principalmente por não influenciar no comportamento de consumo voluntário dos animais. Assim, objetivou-se avaliar doses de $\mathrm{Fe}_{2} \mathrm{O}_{3}$ como marcador fecal por meio de seus efeitos na coloração das fezes de ovinos.

\section{MATERIAL E MÉTODOS}

O trabalho foi aprovado pelo Comitê de Ética em Pesquisa no Uso de Animais (CEUAUFRA, Pará, Brasil; parecer n. 52/2018). Para o estudo foram selecionados quatro ovinos 
machos não castrados, com aproximadamente três meses de idade da raça Santa Inês com peso vivo médio de $33 \pm 3,7 \mathrm{~kg}$.

O delineamento experimental utilizado foi em quadrado latino $4 \times 4$, compreendendo os tratamentos com três doses de adição de óxido de ferro $\left(\mathrm{Fe}_{2} \mathrm{O}_{3}\right)$ de $0,5,10$ e $20 \%$ do concentrado equivalentes a $0,7,5,15$ e $30 \mathrm{~g}^{\text {animal }^{-1}}$, respectivamente. O período experimental teve duração de 47 dias, contabilizando 15 dias para adaptação ao ambiente experimental, sendo que cada subperíodo compreendeu 7 dias mais um de descanso.

O aprisco foi mantido com iluminação artificial no período da noite durante o período experimental de 48 dias a fim de permitir às observações noturnas. Cada gaiola foi dotada de um comedouro principal para fornecimento de feno de capim Tifton-85 triturado, um comedouro secundário para fornecimento do concentrado de milho moído, um bebedouro e um saleiro individual para fornecimento de água e sal ad libitum respectivamente.

As dietas experimentais foram compostas por feno de Tifton 85 moídos e concentrado energético a base de milho moído, numa relação volumoso: concentrado de 84: 16. Os alimentos foram ofertados em cochos individuais, diariamente às $8 \mathrm{e} 17 \mathrm{~h}$, com exceção do primeiro dia de cada subperíodo, em que a dieta foi dividida em 3 partes, sendo a dose de $\mathrm{Fe}_{2} \mathrm{O}_{3}$ misturado ao concentrado total ofertado às 8h, e as demais partes do volumoso, distribuídas as 14 e $20 \mathrm{~h}$. As sobras foram coletadas e pesadas diariamente, para avaliação de consumo pela diferença entre oferta e sobra. $\mathrm{O}$ total de fezes produzida por cada animal foi coletado diariamente às 8 e $17 \mathrm{~h}$, com exceção do primeiro dia de avaliação, em que foram coletadas de 4 em 4 horas a partir do fornecimento dos tratamentos por $24 \mathrm{~h}$.

A caracterização das fezes foi realizada por meio da avaliação da coloração das fezes após a coleta, sendo classificadas pelos avaliadores de acordo com o tom de vermelho préestabelecido com auxílio de tabela de cores com escala de 1 a 4 (Figura 1).

1

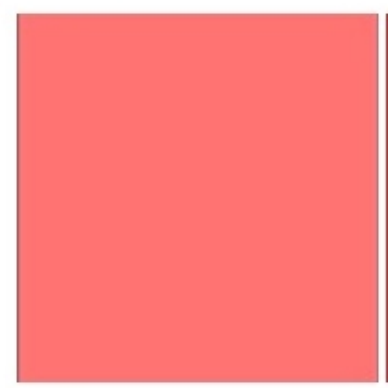

2

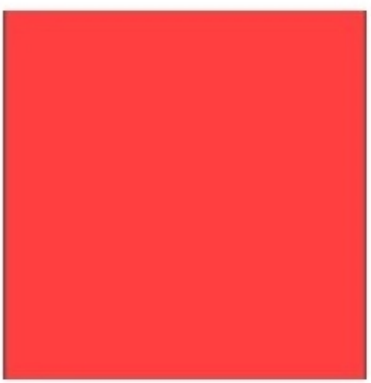

3

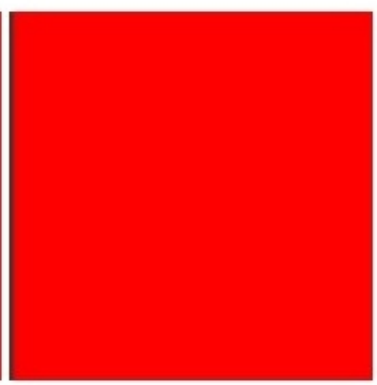

4

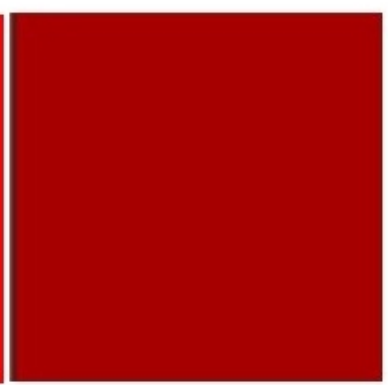

Figura 1. Paleta de cores no tom vermelho para auxiliar a classificação de cor das fezes

Para avaliação da produção fecal, as fezes foram coletadas, pesadas e acondicionadas em sacos plásticos identificados e armazenados em freezer, e posteriormente foram subamostradas para a determinação do teor de matéria seca ao ar (ASE) em estufa de circulação forçada a $65^{\circ} \mathrm{C}$ até atingir peso constante, sendo assim retiradas após $96 \mathrm{~h}$ e pesadas novamente após atingir o equilíbrio com a temperatura ambiente.

No primeiro dia de cada subperíodo foi realizada a avaliação de comportamento por meio da observação visual pontual dos animais de acordo com o etograma (Tabela 1), a cada 5 minutos por um período de 24 horas após o fornecimento do concentrado com o marcador.

O comportamento ingestivo foi monitorado com a contagem do tempo despendido para a ingestão e a mastigação, durante quatro horas em dois períodos após as refeições, entre as 8 
e $12 \mathrm{~h}$ e das 14 às $18 \mathrm{~h}$ para cada tratamento a fim de estimar a eficiência de ruminação, alimentação e tempo de mastigação.

Tabela 1. Etograma elaborado para avaliação comportamental dos ovinos em confinamento após o recebimento dos tratamentos.

\begin{tabular}{cl}
\hline $\begin{array}{c}\text { Atividade } \\
\text { Comportamental }\end{array}$ & \multicolumn{1}{c}{ Descrição } \\
\hline Ócio & $\begin{array}{l}\text { Animal em pé ou deitado que não estava andando, comendo, } \\
\text { ruminando, bebendo água ou em outras atividades. }\end{array}$ \\
\hline Ruminação & $\begin{array}{l}\text { Animal em pé ou deitado, regurgitando, remastigando e redeglutindo } \\
\text { o bolo alimentar. }\end{array}$ \\
\hline Ingestão da dieta & Animal consumindo dieta \\
\hline Ingestão de água & Animal consumindo água \\
\hline Mastigação & Animal em pé ou deitado, mastigando o bolo alimentar. \\
\hline Urinar & Animal expelindo urina \\
\hline Defecar & Animal evacuando fezes do organismo \\
\hline $\begin{array}{c}\text { Interação com a } \\
\text { gaiola }\end{array}$ & Animal mordendo, cheirando, lambendo a estrutura da gaiola \\
\hline Coçar & $\begin{array}{l}\text { Friccionar ou esfregar (a pele) utilizando as patas, boca ou outro } \\
\text { objeto }\end{array}$ \\
\hline Vocalização & $\begin{array}{l}\text { Produção de som como forma de chamamento ou canto, pelo órgão } \\
\text { fonador próprio }\end{array}$ \\
\hline
\end{tabular}

Fonte: Adaptada de (9)

As medidas foram calculadas pela média do tempo despendido de mastigação merícica por bolo ruminal, ingestão da dieta e ruminação, utilizando-se um cronômetro digital, segundo a metodologia descrita por (10), e assim foram determinadas as eficiências de alimentação (EAL) e ruminação (ERU), tempo de mastigação total (TMT) e o número de bolos ruminais $(\mathrm{BOL})$ por meio de equações.

$\mathrm{EAL}=\mathrm{CMS} / \mathrm{TAL}$

em que: EAL ( $\mathrm{g}$ de MS consumida $\mathrm{h}^{-1}$ ) = eficiência de alimentação;

CMS $(\mathrm{g})$ = consumo diário de matéria seca;

$\mathrm{TAL}=$ tempo gasto diariamente em alimentação.

$\mathrm{ERU}=\mathrm{CMS} / \mathrm{TRU}$

em que: ERU ( $g$ MS ruminal $h^{-1}$ ) = eficiência de ruminação;

CMS $(\mathrm{g})=$ consumo diário de matéria seca;

TRU $\left(\mathrm{h} \mathrm{dia}^{-1}\right)=$ tempo de ruminação.

$\mathrm{TMT}=\mathrm{TAL}+\mathrm{TRU}$

em que: TMT (minuto/dia) = tempo de mastigação total.

TAL $=$ tempo gasto diariamente em alimentação.

TRU (h dia-1) = tempo de ruminação.

$\mathrm{BOL}=\mathrm{TRU} / \mathrm{MMtb}$

em que: BOL (no dia-1) = número de bolos ruminais;

TRU $($ h dia-1) $=$ tempo de ruminação.

Ramos PS, Soares BC, Vieira MFA, Tavares AF, Oliveira AJT, Cardoso AA. et al. Potencial do óxido de ferro como marcador fecal em ovinos. Vet. e Zootec. 2021; v28: 001-013. 
MMtb (s bolo-1), tempo de mastigações merícicas por bolo ruminal.

Os dados foram organizados em planilhas de Excel ${ }^{\circledR}$ e avaliados estatisticamente com auxílio do software estatístico SAS versão 9.0 (SAS Institute Inc., Cary, NC, 2004) utilizando como variáveis fixas os tratamentos e tempos de avaliação para algumas variáveis resposta. Após a análise de variância (ANOVA), em caso de verificação de significância das doses de $\mathrm{Fe}_{2} \mathrm{O}_{3}$ ou dos tempos de avaliação, ou até mesmo para a interação entre esses, os dados foram submetidos ao estudo de regressão de primeiro e segundo grau por meio do comando proc GLM avaliando aos níveis de significância de 1 e 5\%.

\section{RESULTADOS}

As doses de $\mathrm{Fe} 2 \mathrm{O} 3$ não apresentaram efeitos no consumo médio diário de feno $(\mathrm{P}=0,8370)$, que foram de 746,$8 ; 746,1 ; 752,5 ; 758,7 \mathrm{~g}$ animal-1 dia-1 para os tratamentos 0,5 , 10 e $20 \%$ respectivamente, e também não houve interação com o tempo após o fornecimento dos tratamentos para essa variável-resposta, portanto a adição dos níveis de $\mathrm{Fe} 2 \mathrm{O} 3$ não afetou o consumo voluntário de massa seca de feno.

$\mathrm{O}$ consumo do concentrado a base de milho não apresentou influência significativa ( $\mathrm{P}$ $=0,3982$ ) das doses de $\mathrm{Fe} 2 \mathrm{O} 3$, que apresentaram consumo do concentrado fornecido com as doses de 0,5 e 10 e $20 \%$ de $\mathrm{Fe}_{2} \mathrm{O}_{3}$ de, respectivamente, 149,9, 149,9, 149,9 e 147,5 g animal1 dia-1.

Os seis dias decorrentes de avaliação dos subperíodos após o fornecimento do marcador apresentou efeito sob o consumo voluntário de feno, que decresceu linearmente ao longo dos dias de coleta (Tabela 2).

Tabela 2. Consumo médio diário de feno e concentrado e produção fecal na base da matéria seca nos dias após aplicação dos tratamentos com $\mathrm{Fe}_{2} \mathrm{O}_{3}$

\begin{tabular}{cccc}
\hline \multirow{2}{*}{$\begin{array}{c}\text { Dias após aplicação } \\
\text { dos tratamentos }\end{array}$} & Feno & concentrado & Fezes \\
\cline { 2 - 4 } $\mathrm{g} \mathrm{dia}^{-1}$ \\
\hline 1 & $771,3 \pm 44,5$ & $146,3 \pm 14,7$ & $80,91 \pm 31,24$ \\
2 & $758,6 \pm 43,9$ & $149,9 \pm 0,07$ & $109,99 \pm 41,92$ \\
3 & $774,8 \pm 41,9$ & $149,9 \pm 0,07$ & $91,69 \pm 34,69$ \\
4 & $713,1 \pm 89,4$ & $149,9 \pm 0,07$ & $138,81 \pm 60,12$ \\
5 & $758,8 \pm 38,3$ & $149,9 \pm 0,04$ & $124,12 \pm 54,39$ \\
6 & $722,3 \pm 78,0$ & $149,9 \pm 0,07$ & $124,12 \pm 44,89$ \\
\hline P (valor) & 0,0367 & 0,4276 & 0,0205 \\
\hline Equação & $\mathrm{y}=-8,7457 \mathrm{x}+780,43$ & - & $\mathrm{y}=25,886 \mathrm{x}+207,73$ \\
\hline CV(\%) & 8,4 & 4,0 & 45,5 \\
\hline
\end{tabular}

Não houve efeito dos tratamentos na produção fecal $(\mathrm{P}=0,6120)$ com 101,0, 112,2, 107,3 e 120,6 $\mathrm{g} \mathrm{dia}^{-1}$ respectivamente para $0,5,10$ e $20 \%$ do $\mathrm{Fe}_{2} \mathrm{O}_{3}$, mas por outro lado houve crescimento linear desta variável ao longo dos dias de coleta (Tabela 2).

As variáveis de comportamento ingestivo não foram influenciadas $(\mathrm{P}>0,05)$ pela inclusão de doses de $\mathrm{Fe}_{2} \mathrm{O}_{3}$ na dieta (Tabela 3).

Ramos PS, Soares BC, Vieira MFA, Tavares AF, Oliveira AJT, Cardoso AA. et al. Potencial do óxido de ferro como marcador fecal em ovinos. Vet. e Zootec. 2021; v28: 001-013. 
Em 24 horas de avaliação, as porcentagens do tempo despendido com as atividades de comportamento também não apresentaram efeito dos tratamentos (Figura 2), sendo que os animais passaram a maior parte do tempo na atividade de ócio, em média $35 \%$ do tempo avaliado, seguida pela atividade de ruminação ( $32 \%$ do tempo).

Tabela 3. Valores das variáveis do comportamento ingestivo em 24 após a aplicação dos tratamentos com $\mathrm{Fe}_{2} \mathrm{O}_{3}$.

\begin{tabular}{ccccccc}
\hline \multirow{2}{*}{ Doses } & 0 & 5 & 10 & 20 & $\mathrm{CV}(\%)$ & $\mathrm{p}$ (valor) \\
\cline { 2 - 7 } & 909,50 & 914,25 & 919,75 & 927,00 & 5,30 & 0,6067 \\
CMS $\left(\mathrm{g} \mathrm{dia}^{-1}\right)$ & 1,91 & 2,04 & 2,44 & 1,97 & 18,90 & 0,5396 \\
TAL $\left(\mathrm{h} \mathrm{dia}^{-1}\right)$ & 1,52 & 1,70 & 1,68 & 1,50 & 19,40 & 0,8933 \\
TRU $\left(\mathrm{h} \mathrm{dia}^{-1}\right)$ & 53,25 & 49,25 & 61,00 & 46,50 & 20,40 & 0,9205 \\
MMtb $\left(\mathrm{s} \mathrm{bolo}^{-1}\right)$ & 519,94 & 452,01 & 384,72 & 480,58 & 24,30 & 0,4724 \\
EAL $\left(\mathrm{g} \mathrm{MS} \mathrm{h}^{-1}\right)$ & 513,11 & 551,55 & 570,20 & 625,69 & 17,90 & 0,8156 \\
ERU $\left(\mathrm{g} \mathrm{MS} \mathrm{h}^{-1}\right)$ & 206,4 & 224,4 & 247,2 & 207,6 & 13,30 & 0,6964 \\
TMT $\left(\mathrm{m} \mathrm{dia}^{-1}\right)$ & 103 & 124 & 99 & 116 & 4,0 & 0,9316 \\
BOL $\left(\mathrm{n}^{\mathrm{o}} \mathrm{dia}^{-1}\right)$ & & & & & & \\
\end{tabular}

Consumo de massa seca (CMS), tempo de alimentação (TAL), tempo de ruminação (TRU), tempo de mastigações merícicas por bolo ruminal (MMtb), eficiência alimentar (EAL), eficiência ruminal (ERU), tempo de mastigação total (TMT) e número de bolos ruminais (BOL).

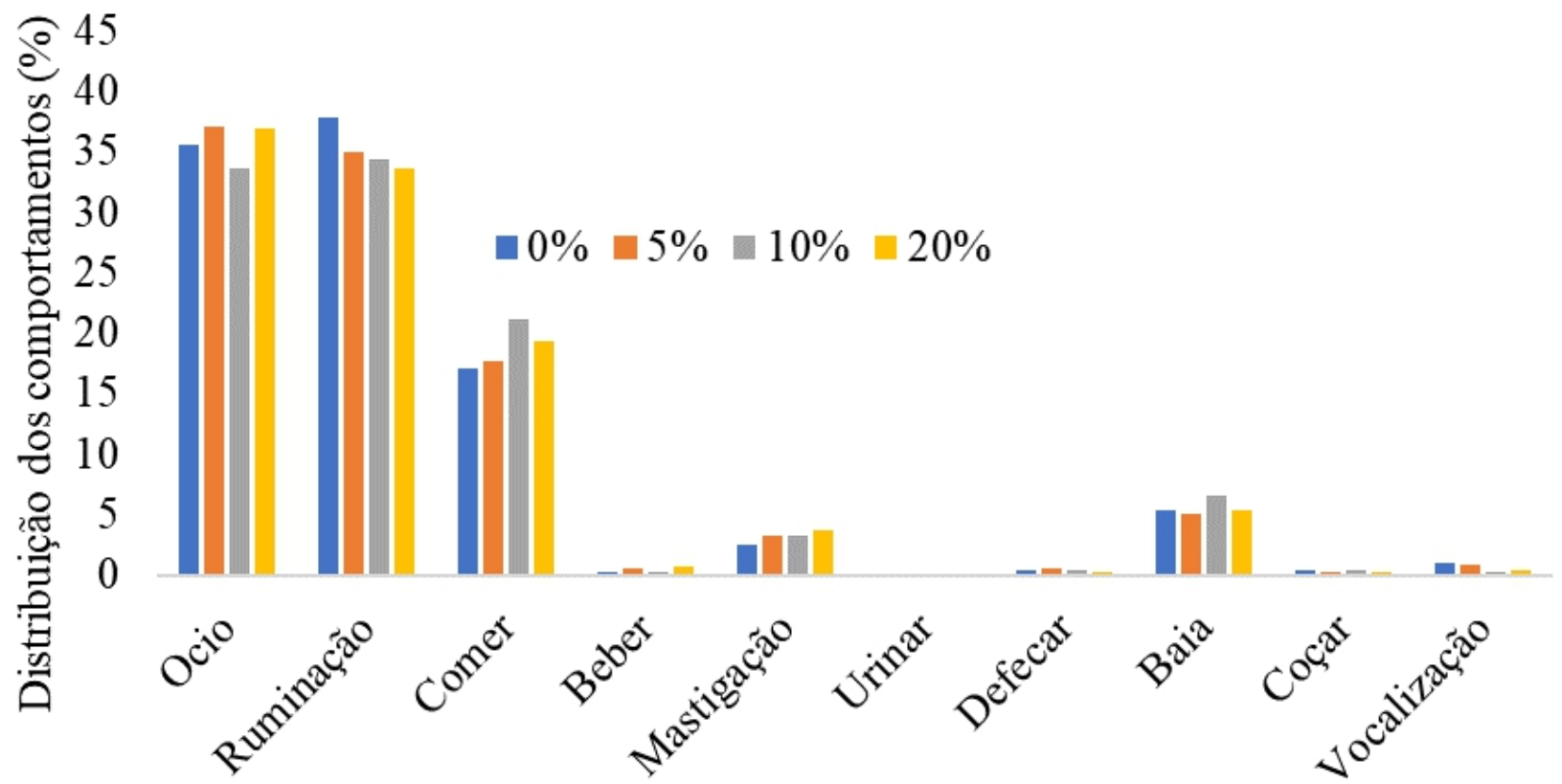

Figura 2. Distribuição das atividades comportamentais ao longo das 24 h após aplicação dos tratamentos. 
A coloração das fezes apresentou efeito das doses de $\mathrm{Fe}_{2} \mathrm{O}_{3}(\mathrm{p}<0,0001)$, do tempo após o fornecimento dos tratamentos $(p<0,0001)$ e da interação entre doses e tempo $(p=0,0234)$ (Figura 3).

$\mathrm{O}$ tratamento que recebeu a dose $20 \%$ de $\mathrm{Fe}_{2} \mathrm{O}_{3}$ foi o primeiro a apresentar as fezes com intensidade da cor vermelha (Figura 4), que ocorreu na coleta de $16 \mathrm{~h}$ após o fornecimento do $\mathrm{Fe}_{2} \mathrm{O}_{3}$.
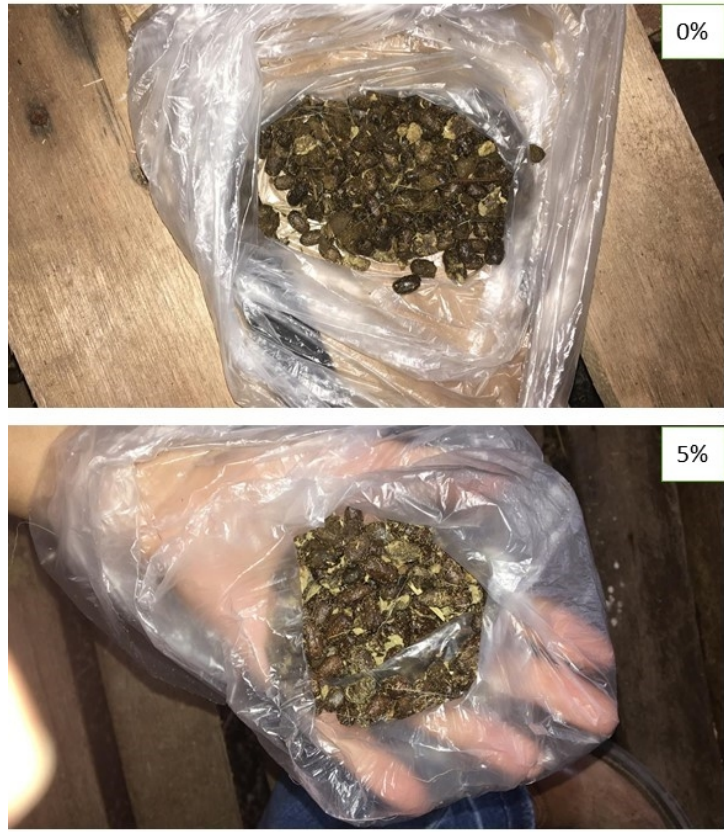
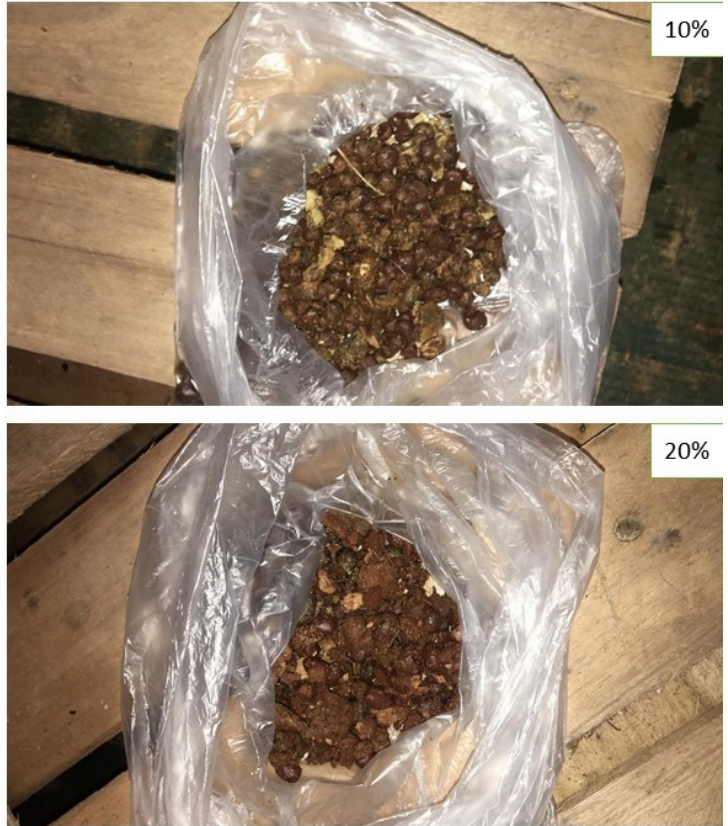

Figura 3. Coloração das fezes às 28 h após a ingestão dos tratamentos.

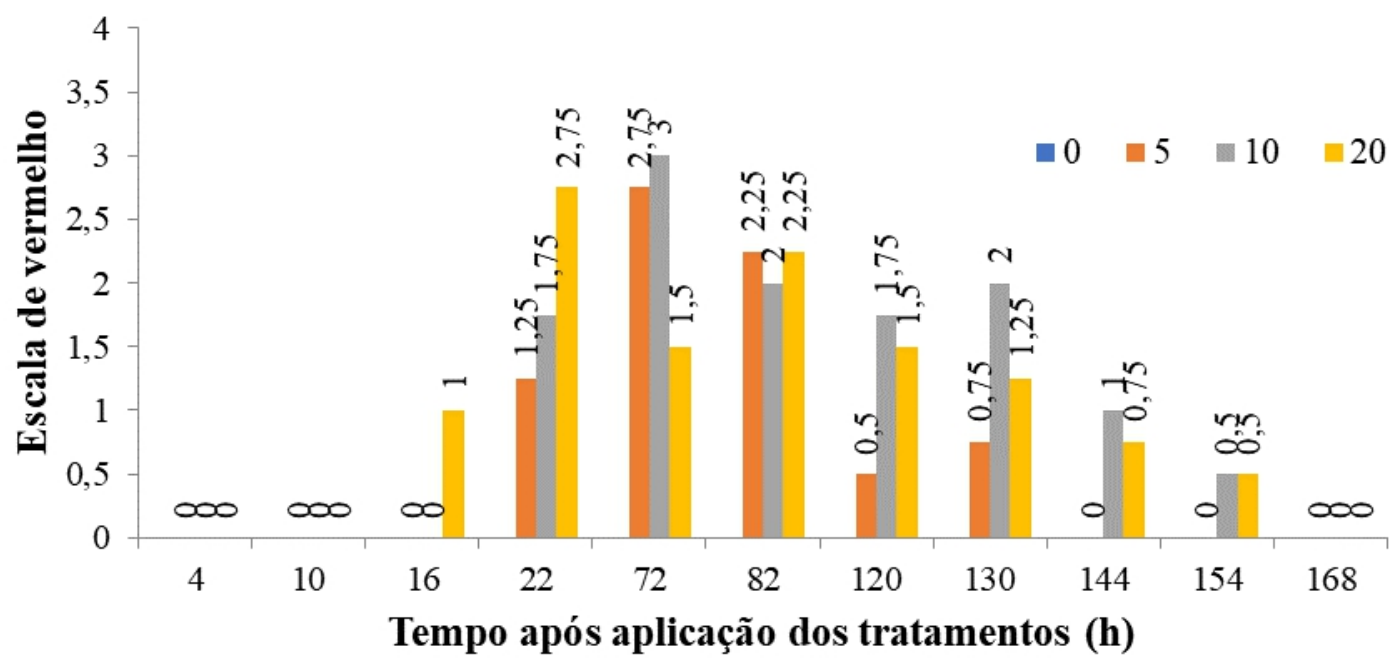

Figura 4 - Coloração das fezes ao longo do tempo após aplicação dos tratamentos

\section{DISCUSSÃO}

A ausência de efeitos no consumo do concentrado (Tabela 2) indicou que adição do $\mathrm{Fe}_{2} \mathrm{O}_{3}$ junto a suplementação concentrada pode ser uma alternativa viável para o consumo

Ramos PS, Soares BC, Vieira MFA, Tavares AF, Oliveira AJT, Cardoso AA. et al. Potencial do óxido de ferro como marcador fecal em ovinos. Vet. e Zootec. 2021; v28: 001-013. 
total do mesmo como marcador, sem a necessidade de forçar a ingestão pelos animais, o que poderia acarretar estresses e mudanças comportamentais.

A ausência de efeitos no consumo de feno demonstrou a possibilidade de uso deste produto como marcador em experimentos com suplementação a pasto, sem influência no consumo de massa seca dos animais. A redução do consumo voluntário de feno ao longo do tempo (Tabela 2) se ajustou ao modelo linear decrescente, embora tenha apresentado baixo coeficiente de determinação, provavelmente essa ingestão tenha sido reflexo de fatores fisiológicos diante do fornecimento do volumoso, ou seja, pelas exigências energéticas do animal ou pelos moduladores psicogênicos.

A baixa palatabilidade pode ser um limitante para o consumo do marcador, mesmo misturado a ingredientes da dieta. Conforme Gomes et al. (11), a baixa palatabilidade, e até a digestibilidade, podem afetar diretamente na efetividade da mastigação e ruminação e, consequentemente, perdas de produtividade. No entanto, a ausência de efeitos no consumo (Tabela 2) e no comportamento de ingestão (Tabela 3) sugerem a ausência de efeito negativo da palatabilidade do $\mathrm{Fe}_{2} \mathrm{O}_{3}$ para os ovinos.

A ausência de efeitos dos tratamentos no total de fezes demonstrou-se um indicativo de que o $\mathrm{Fe}_{2} \mathrm{O}_{3}$ pode ser utilizado como marcador sem afetar a ingestão e a digestão dos alimentos pelo ruminante. Por outro lado, a variação no total de fezes ao longo do tempo se ajustou em modelo linear crescente, possivelmente como consequência da variação no consumo.

A semelhança no consumo entre os tratamentos pelo $\mathrm{Fe}_{2} \mathrm{O}_{3}$, considerando que a dieta foi a mesma, pode justificar a não diferenciação dos parâmetros comportamentais relacionados a ingestão, como o TAL e TRU entre outros, e consequentemente, a eficiência de alimentação (EAL $\mathrm{g} \mathrm{MS}^{-1} \mathrm{~min}^{-1}$ ) e de ruminação $\left(\right.$ ERU $\mathrm{g} \mathrm{MS}^{-1} \mathrm{~min}^{-1}$ ) (Tabela 3).

A eficiência de ruminação ou mastigação pode ser reduzida em dietas com maiores proporções de concentrado ou com uso de alimentos triturados, e a redução na eficiência de ruminação não pode ser compensada pelo prolongamento da atividade de ruminação, porém mesmo com diferentes dietas, não houve diferenças entre as variáveis de eficiência de alimentação e ruminação na análise de comportamento ingestivo de ovinos Bürger et al. (10), Carvalho et al. (13), Pinheiro et al. (14) e Fontenele et al. (15).

$\mathrm{O}$ número de bolos ruminados $\left(\mathrm{n}^{\mathrm{o}} \mathrm{dia}^{-1}\right)$ e o tempo de mastigações bolo ${ }^{-1}$ (s) e, consequentemente, tempo de mastigação total $\left(\min \operatorname{dia}^{-1}\right)$ não foram influenciados pelos tratamentos $(\mathrm{P}>0,05)$. Segundo (12), o número de bolos ruminais e o tempo gasto na mastigação total e por bolo, são diretamente proporcionais ao tipo do alimento e a quantidade de fibra, no entanto o $\mathrm{Fe}_{2} \mathrm{O}_{3}$ não alterou a qualidade do alimento.

O número de bolos ruminados por dia depende do tempo de ruminação e do tempo gasto para ruminar cada bolo de acordo com Cirne et al. (16), o que explica o fato de não ter ocorrido variação, já que não houve diferença na alimentação. Pinheiro et al (14) e Mendes et al. (17), também não encontraram efeito de diferentes dietas sobre tempo gasto por bolo ruminado, nem nos números de mastigações por bolo, número de bolos ruminados $\operatorname{dia}^{-1}$ e no tempo total de mastigação, mas conforme Macedo et al. (18), as variáveis que afetam o comportamento possibilitam a melhoria no desempenho quando se faz ajustes no manejo alimentar dos animais.

As atividades comportamentais com maiores distribuições foram a de ócio, ruminação, alimentação e de interação com a gaiola (Figura 2). Este último comportamento pode indicar desconforto dos animais devido à restrição de espaço das gaiolas, não havendo relação com os 
tratamentos e sim com o ambiente. Embora o risco de erros deste tipo de análise, estes foram reduzidos com avaliações em intervalos curtos de tempo, pois de acordo com Marques et al. (19), a determinação de uma escala de tempo entre as observações, pode comprometer os resultados das avaliações.

A coloração nas fezes foi observada em todos os tratamentos com $\mathrm{Fe}_{2} \mathrm{O}_{3}$ a partir das 22 $\mathrm{h}$ após a aplicação dos tratamentos possibilitando distinguir os tratamentos por intensidades nos tons de vermelho, que permaneceram em todos os tratamentos com a adição de $\mathrm{Fe}_{2} \mathrm{O}_{3}$ até $130 \mathrm{~h}$ após o fornecimento do marcador (Figura 4).

Apenas uma exceção foi observada no tratamento com $20 \%$ de $\mathrm{Fe}_{2} \mathrm{O}_{3}$ em que um animal demonstrou coloração das fezes a partir das 16 h, o que mesmo na média, apresentou o efeito deste tratamento devido ao intenso tom de vermelho observado (Figura 4).

Embora observado em apenas uma unidade experimental, essa dinâmica pode indicar a possibilidade de maior velocidade de passagem do marcador quando em alta concentração, e consequentemente sugerindo a possibilidade deste produto ter pigmentado o bolo fecal de refeições anteriores ao seu fornecimento, uma vez que o consumo de matéria seca e o quantitativo fecal não diferiram. Segundo (12), o aumento no consumo eleva o tempo de alimentação e reduz o tempo de ruminação.

Ao longo do tempo a intensidade dos tons de vermelho presente nas fezes sob os tratamentos com $\mathrm{Fe}_{2} \mathrm{O}_{3}$ foram diminuindo, e a dose de 5\% foi a primeira a demonstrar ausência de percepção de coloração nas fezes, isso ocorreu após $144 \mathrm{~h}$ da ingestão do marcador, enquanto as doses 10 e $20 \%$ persistiram até $154 \mathrm{~h}$ de avaliação (Figura 4).

$\mathrm{Na}$ coleta as $168 \mathrm{~h}$ após a ingestão do $\mathrm{Fe}_{2} \mathrm{O}_{3}$ correspondendo ao $7^{\circ}$ dia experimental, todos os animais apresentaram fezes sem pigmentação aparente (Figura 4), possivelmente demonstrando o final da excreção do conteúdo ingerido no dia da aplicação. No entanto, é possível inferir que a intensidade da coloração e o tempo de liberação de fezes pigmentadas pode depender também da digestibilidade do alimento e da intensidade de ruminação.

Dentre os níveis de $\mathrm{Fe}_{2} \mathrm{O}_{3}$ avaliados, o tratamento com $10 \%$ demonstrou alta capacidade e persistência em pigmentar as fezes, sua persistência na coloração poderia permitir menores riscos para avaliações de taxa de passagem em alimentos de menor digestibilidade. Com o uso do $\mathrm{Fe}_{2} \mathrm{O}_{3}$ como marcador em suplementos seria possível identificar as fezes de animais em áreas como confinamentos ou em pasto sem a necessidade de forçar a ingestão do marcador, e assim facilitar a coleta de fezes sem alteração no comportamento.

Ao avaliar os marcadores internos e externos (20), constatou que alguns são fornecidos embalados em cápsulas de papel ou gel ou inseridos no animal pela cânula, necessitando de procedimentos estressantes ou cirúrgicos aos animais. Enquanto com o $\mathrm{Fe}_{2} \mathrm{O}_{3}$ não há necessidade de procedimentos invasivos, apenas sua mistura com um alimento ou ingrediente de alta palatabilidade, a incorporação dos níveis de $\mathrm{Fe}_{2} \mathrm{O}_{3}$ em 75 g de concentrado a base de milho foi o suficiente para colorir o bolo alimentar e refletir nas fezes.

A capacidade de interação do $\mathrm{Fe}_{2} \mathrm{O}_{3}$ com todo o bolo alimentar, embora tenha sido fornecido em apenas um dos ingredientes da dieta, mostrou-se positiva, no entanto há necessidade de mais estudos sobre suas interações químicas e físicas podendo possibilitar seu uso para avaliação de taxa de passagem com maior precisão.

(21) observou diferença entre marcadores na determinação da taxa de passagem, relatando que o cromo apresentou maior taxa de passagem em relação ao lantânio (La) e o itérbio $(\mathrm{Yb})$, fato que pode ser explicado pelo método de incorporação na dieta, pois nesse 
caso o La e o $\mathrm{Yb}$ foram incorporados ao milho, diferente do cromo que foi misturado em toda ração.

No entanto (22) relatou que o tempo necessário para a excreção de um marcador depende da condição da dieta e do tipo de animal, e em ovinos e bovinos é necessário um período de adaptação de 6 a 8 dias antes do período experimental.

A coloração das fezes facilitaria a realização de estudos, não somente de estimativa fecal, mas para a localização destas no ambiente produtivo e até mesmo para a verificação da dispersão de moléculas, organismos e sementes pelas fezes, porém o uso desta técnica ainda merece maiores investigações, principalmente analíticas, verificando os efeitos do óxido de ferro na microbiota ruminal e a possibilidade de quantificação nas fezes, que possibilitaria seu uso como estimador.

A ausência de mudanças no comportamento dos animais e a facilidade de consumo junto aos alimentos, assim como sua efetividade na identificação das fezes sugere o potencial do $\mathrm{Fe}_{2} \mathrm{O}_{3}$ como marcador. Rodriguez et al. (23) confirmaram as vantagens dos marcadores comparados aos processos invasivos, que causam mudanças comportamentais ou físicas nos animais, tendo em vista a necessidade de utilização de cânulas reentrantes no trato digestivo, sacolas de coleta de fezes e até mesmo esvaziamento do trato digestivo ou abate dos animais. Segundo Aroeira et al. (24) a estimativa da produção fecal com o uso de marcadores favorece as avaliações, mesmo que os animais apresentem diferenças entre si.

\section{CONCLUSÃO}

$\mathrm{O}$ óxido de ferro $\left(\mathrm{Fe}_{2} \mathrm{O}_{3}\right)$ apresenta potencial como marcador fecal para ruminantes, tornando-se uma ferramenta para auxiliar em pesquisas de produção animal por meio de seus efeitos na coloração das mesmas, sem alteração no comportamento de consumo dos animais.

$\mathrm{O}$ nível de $10 \%$ de $\mathrm{Fe}_{2} \mathrm{O}_{3}$ no suplemento, equivalente a $15 \mathrm{~g}$ animal ${ }^{-1}$ em ovinos, apresentou maior persistência visual na pigmentação das fezes.

\section{REFERÊNCIAS}

1. Canesin RC, Fiorentini G, Berchielli TT. Inovações e desafios na avaliação de alimentos na nutrição de ruminantes. Rev Bras Saude Prod Anim. 2012;13(4):938-53.

2. Mertens DR. Regulation of forage intake. In: Mertens DR. National conference on forage quality. Evaluation and utilization. Lincoln: University of Nebraska; 1994. p.450-93.

3. Eloy LR, Rocha MG, Pötter L. Consumo de forragem por novilhas de corte recebendo farelo de arroz com e sem ionósforo. Cienc Rural. 2014; 44: 1223-1228.

4. Berchielli TT, Garcia AV, Oliveira SG. Principais técnicas de avaliação aplicadas em estudo de nutrição. In: Berchielli TT, Garcia AV, Oliveira SG. Nutrição de ruminantes. Jaboticabal: FUNEP; 2011. p.565-600.

5. Salman AK, Ferreira ACD, Soares JPG, Souza JP. Metodologia para avaliação de alimentos para ruminante doméstico. Porto Velho: EMBRAPA Rondônia; 2010. 
6. Ítavo LCV, Valadares Filho SC, Silva FF, Valadares RFD, Paulino MF, Ítavo CCBF, et al. Comparação de indicadores e metodologia de coleta para estimativas de produção fecal e fluxo de digesta em bovinos. Rev Bras Zootec. 2002;31(4):1833-9.

7. Figueiredo MRP. Indicadores externos de digestibilidade aparente em ovinos [dissertação]. Belo Horizonte: Escola de Veterinária, Universidade Federal de Minas Gerais; 2011.

8. Vieira, MS, Vieira AA. Determinação do tempo de abate de suínos em ensaios de digestibilidade pelo método do sacrifício. In: Anais do 9o Encontro Latino Americano de Pós-Graduação; 2009; São José dos Campos. São José dos Campos: Universidade do Vale do Paraíba; 2009.

9. Paranhos da Costa MJR, Cromberg VU. Relações materno-filiais em bovinos de corte nas primeiras horas após o parto. In: Comportamento Materno em Mamíferos: bases teóricas e aplicações aos ruminantes domésticos, São Paulo: Sociedade Brasileira de Etologia, 10, 1998. p.215-235.

10. Bürger PJ, Pereira JC, Queiroz AC, Silva JFC, Valadares Filho SC, Cecon PR, Casali ADP. Comportamento ingestivo em bezerros holandeses alimentados com dietas contendo diferentes níveis de concentrado. Rev Bras Zootec. 2000;29(1):236-42.

11. Gomes SP, Borges ALCC, Borges I, Macedo Júnior GL, Silva AGM, Pancoti CG. Efeito do tamanho de partícula do volumoso e da frequência de alimentação sobre o consumo e a digestibilidade em ovinos. Rev Bras Saude Prod Anim. 2012;13(1):137-49.

12. Van Soest PJ. Nutritional ecology of the ruminant. 2nd ed. Ithaca: Cornell University Press; 1994.

13. Carvalho CGGP, Pires AJV, Silva FF, Veloso CM, Silva RR, Silva HGO, et al. Comportamento ingestivo de cabras leiteiras alimentadas com farelo de cacau ou torta de dendê. Pesqui Agropecu Bras. 2004;39:919-25.

14. Pinheiro AA, Veloso CM, Rocha Neto AL, Silva RR, Silva FF, Mendes FBL, et al. Comportamento ingestivo de novilhas leiteiras alimentadas com níveis de farelo de cacau (Theobroma cacao) na dieta. Rev Bras Saude Prod Anim. 2012;13(1):224-36.

15. Fontenele RM, Pereira ES, Carneiro MSS, Pimentel PG, Cândido MJD, Regadas Filho JGL. Consumo de nutrientes e comportamento ingestivo de cordeiros da raça Santa Inês alimentados com rações com diferentes níveis de energia metabolizável. Rev Bras Zootec. 2011;40(6):1280-6.

16. Cirne LGA, Sobrinho AGS, Santana VT, Silva FU, Lima NLL, Oliveira EA, et al. Comportamento ingestivo de cordeiros alimentados com dietas contendo feno de amoreira. Semin Cienc Agrar. 2014;35(2):1051-60 
17. Mendes FBL, Silva FF, Silva RR, Carvalho GGP, Cardoso EO, Rocha Neto AL, et al. Avaliação do comportamento ingestivo de vacas leiteiras em pastejo de Brachiaria brizantha recebendo diferentes teores de concentrado na dieta. Semin Cienc Agrar. 2013;34(6):2977-90.

18. Macedo CAB, Mizubuti IY, Moreira FB, Pereira ES, Ribeiro ELA, Rocha MAR, et al. Comportamento ingestivo de ovinos recebendo dietas com diferentes níveis de bagaço de laranja em substituição à silagem de sorgo na ração. Rev Bras Zootec. 2007;36(6):191016.

19. Marques JÁ, Pinto AP, Abrahão JJS, Nascimento WGN. Intervalo de tempo entre observações para avaliação do comportamento ingestivo de tourinhos em confinamento. Semin Cienc Agrar. 2008;29(4):955-60.

20. Longo TR. Avaliação de marcadores externos e internos para estimar excreção fecal e consumo em ovinos alimentados com pastagem natural ou feno de tifton 85 [dissertação]. Santa Maria: Centro de Ciências Rurais, Universidade Federal de Santa Maria; 2015.

21. Oetting LL. Avaliação de diferentes marcadores para a determinação da digestibilidade e taxa de passagem do alimento em suínos [dissertação]. Piracicaba: Centro de Energia Nuclear na Agricultura, Universidade de São Paulo; 2002.

22. D'Mello JPF. Use of markers. In: Marais JP. Farm animal metabolism and nutrition. Wallingford: CAB International; 2000. p.255-77.

23. Rodríguez NM, Saliba EOS, Guimarães Júnior R. Uso de indicadores para a estimativa de consumo a pasto e digestibilidade. In: Anais do Simpósio da $43^{\circ}$ Reunião Anual da SBZ. 2006 João Pessoa: Sociedade Brasileira de Zootecnia; 2006, p.263-282.

24. Aroeira LJM, Lopes FCF, Soares JPG. Daily intake of lactating crossbred cows grazing elephantgrass rotationally. Pesquisa Agropecuária Brasileira. 2001; 36(6): 911-917.

Recebido em: 12/10/2020 Aceito em: 03/03/2021 